\title{
Three Cases with Artifacts Presented in the Form of Intensive Diffuse Accumulation in the Liver and Spleen After Application of 99mTcMDP
}

\author{
Luljeta Abdullahu ${ }^{1}$, Armend Jashari ${ }^{1}$, Ylli Kaçiu ${ }^{1}$ and Naser Gjonbalaj ${ }^{2}$ \\ ${ }^{1}$ Department of Nuclear Medicine, University Clinical Center of Kosovo "UCCK”, Prishtina \\ ${ }^{2}$ Clinic of Radiology University Clinical Center of Kosovo “UCCK”, Prishtina \\ *Corresponding Author: Luljeta Abdullahu, Department of Nuclear Medicine, \\ University Clinical Center of Kosovo "UCCK”, Prishtina
}

Received: June 25, 2020

Published: September 25, 2021

(C) All rights are reserved by Luljeta

Abdullahu., et al.

\begin{abstract}
The literature refers to various cases of non osseous uptake of 99mTc-MDP , and various examples are presented about radiobio distribution problems of osteotropic radiopharmaceuticals, which may be unpredicted or altered, for which it is not it is always possible to conclude that this radiopharmaceutical (this agen $\mathrm{t}$ ) can be absorbed in areas far from active bone formations where calcification is not present.

Purpose: We report 3 cases with intensive diffuse uptake of 99mTc-MDP in the liver and spleen in patients with primary breast tumor, lung tumor and a suspected case of osteomyelitis, brought to our department, with the aim of detecting metastases in the skeleton respectively diagnosing the primary process to an adolescent's on left ankle. The aim is to interpret / refer to and discuss these cases with detected artifacts that exhibit difficulties in identifying soft tissue etiologies, organized according to the uptake mechanisms of $99 \mathrm{mTc}-\mathrm{MDP}$.

Discussion: One of the impurities that can sometimes be observed in the eluent samples from the generators is Aluminium, in addition to the reduced $99 \mathrm{mTc}$ hydrolysis process, also due to the excessive amount of Al3 which would cause the particles to form as a digestion "colloid" which, if injected, will be localized in the liver and spleen.

Impression: In my experience almost 20 years ago, this is the first time I've had the chance to see such an artifact, such as intensive diffuse uptake of liver and spleen, along with the $99 \mathrm{mTc}$-MDP accumulation on skeleton, until in the literature I had the opportunity to see and read about the various causes of this phenomenon, such as these presented cases, we would like to share this experience with you with presented facts. How much other artifacts like focal accumulation in the liver, lung, amyloidosis of the heart and unusual or common accumulation in soft tissues and other organs outside bone tissue have been the most different.
\end{abstract}

Keywords: 99mTc MDP; 99mTc Generator; Colloid Particles; Liver; Spleen; Skeleton; Siemens-E-Cam Dual Head Gamma Camera

\section{Case Presentation}

An artifact characterized by abnormal accumulation of osteotropic radiopharmaceutical, absorbed in areas far from active bone formations where calcification is not present, with the consequences of unforeseen imaging manifestations in a bone scan, arouses the curiosity of the cause of the mechanism of non-com- mon bio distribution of 99mTc-MDP.

The modified bio distribution of the $99 \mathrm{mTc}$ labeled radiopharmaceutical is usually associated with increased amounts of $99 \mathrm{mTc}$ radiochemical impurities, such as $99 \mathrm{mTc0} 4$ free and particle form impurities such as $99 \mathrm{mTc}$ colloids or the hydrolysis process of 99mTc reduced. 
The authors have presented five possible physicochemical etiologic factors that may affect the pathway of bio distribution of the radiopharmaceutical:

- $\quad$ Preparation and formulation of the radiopharmaceuticals

- $\quad$ Problems caused by the techniques and procedures of administration of the radiopharmaceutical

- Factors caused by pathophysiological and biochemical changes

- $\quad$ From previously performed medical procedures, such as surgical, radiation therapy and dialysis; and

- Undesirable effects and interactions vith other medicinal products.

\section{Introduction}

Knowledge of transport mechanisms and local localization of a given radiopharmaceutical is essential to understanding different factors responsible for changing and bio distributing a radiopharmaceutical.

In order to understand the basis and the mechanisms involved in changing the bio distribution of a radiopharmaceutical, the first criterion is the Radiopharmaceutical quality control test, the physical and chemical evaluation, chemical and biological purity.

\section{Presence of impurity}

We know that some form of radiopharmaceutical should have the desired radionuclide amounts and other radionuclide contaminants (Pure Radionuclide), some radiochemical impurities, which in minimal quantities are allowed in radionuclide amounts. But as a result of undesirable chemical reactions, or even due to radiolysis, unacceptable amounts of radiochemical impurities in a radio-pharmaceutical preparation would result in a change in bio distribution and poor image quality.

The components that contribute to the quality of the $99 \mathrm{mTc}$ eluate are: either.

The $99 \mathrm{mTc}$ eluate may have the presence of some constituents:

\section{Radionuclide purity}

In the $99 \mathrm{~m}$ Tc product extracted (eluated) from the generator, there will always be a trace of molybdenum, but always in the minimum allowed amount of radionuclides.
Chemical impurities

Aluminum ions can adversely affect some radioactive preparations that cause unexpected accumulation of activity (for example, accumulation in the lungs of the material prepared for bone imaging).

Very low concentrations of $\mathrm{Al} 3(1.0 \mu \mathrm{g} / \mathrm{mL})$ can react with buffer phosphates by forming unstable aluminum phosphates, and generated particles can block lung capillaries.

The higher concentrations of $\mathrm{Al} 3(10 \mu \mathrm{g} / \mathrm{mL})$ may react with 99mTc-MDP that form intractable particles, which are obtained from ERS cells in the liver (figures presented) and spleen.

Radiochemical impurity, or undesirable chemical form of 99mTc-The expected chemical form for the $99 \mathrm{mTc}$ eluent from the generator is - the pertechnetate $\mathrm{TcO} 4$ - this ion will be reduced to a lower oxidation state before it binds to the corresponding ligand to form the final dose of human administration radiopharmaceutical. Any $99 \mathrm{mTc}$ that is not in the form of pertechnetate is considered a radiochemical impurity.

The $99 \mathrm{mTc}$ valence reduction from 7 to 3 or 5 valence is achieved with the use of a reducing agent such as stannous chloride (Sn2), where reduced $99 \mathrm{mTc}$ is chemically more reactive and form complexes with a wide variety of chelating agents such as MDP methylene diphosphonate, DTPA, MAG3, and sestamibi.

But Sn2's reduction capacity may be drastically reduced by a variety of factors, such as oxidation during storage and preparation of the kit.

So the optimal Sn2 levels in kit reagents are necessary to minimize 99mTc impurities.

The impurity that is sometimes observed in the samples extracted from the $99 \mathrm{mTc}$ generator is hydrolysis of $99 \mathrm{mTc}$ reduced. This material occurs as an "insoluble colloid", which, if injected, will be localized in the liver and spleen. The presence of colloid can be easily determined by chromatographic procedures.

Hydrolysis with excess stannous ions can result in the formation of $99 \mathrm{mT}$ c colloids, which can then be obtained by ERS.

\section{Radiolysis products}

The presence of reactive radiolabel products capable of oxida- 
tion may interfere with the chemical processes required to make radiopharmaceuticals.

\section{Method of work}

During one normal working day, were done a total of 18 bone scans per day, in these last three patients, the application of the radiopharmaceutical was done after the second label, which was done nearly 2 hours after the usual 99-Mo generator elution, usually the first elution starts at 7 o'clock , $15 \mathrm{~min}$ in the morning, due to lack of activity in the first compound for the realization of bone scan previously determined, the preparation of the new ligand was done according to the usual protocol.

Bone scan (whole body scan) was performed with Siemens-ECam dual head Gamma camera, 3-4 hours after application of the diagnostic dose of $99 \mathrm{mTc}$-MDP radiopharmaceutical.

In our department we were able to see three recent cases with intense 99mTc-MDP diffuse liver and spleen accumulations, for which we are not exactly sure which mechanism has caused the creation of these colloid particles, as a non-specific finding, we began to look for the cause of the findings of the conditions which have exactly caused this mechanism.

Since we lacked a chromatograph to verify the quality control of the compound labeled and to verify the overcoming presence of possible radio-chemical impurities, given that in the last 3 cases the application of the radiopharmaceutical was made after second marking, which is done in less than 2 hours after the usual 99mTc generator elution, which usually starts at 7:15 am in the morning due to the lack of activity in the first compound for the realization of previously designated bone scan, a new pharmaceutical was prepared according to the usual protocol.

The last three out of the total 18 patients that were labeled late have shown intense diffuse accumulation at the liver and spleen levels, in addition to the usual accumulation of skeleton (Figure $1-4)$.

The history of the disease, any possible accompanying disease, laboratory tests, the use of medications and their possibility of interacting has been reviewed for each case, none of the patients have previously conducted any RM research or even were not in hemodialysis, and all three patients did not have any significant data that would suggest the cause of this performance.

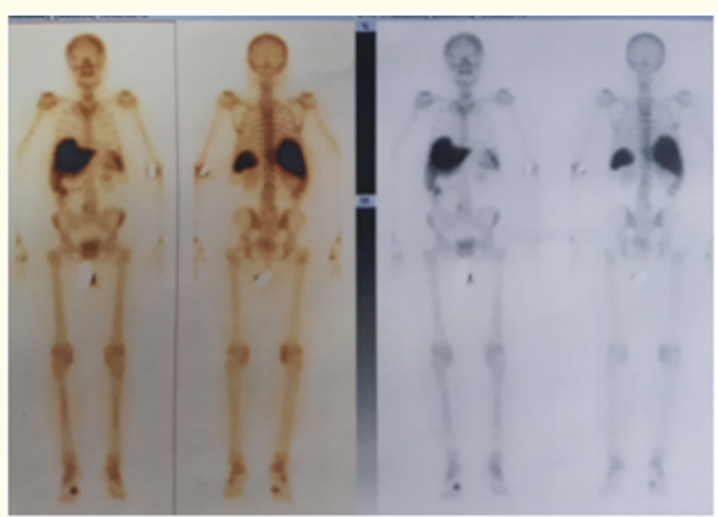

Figure 1: Pat. with Ca mammae.

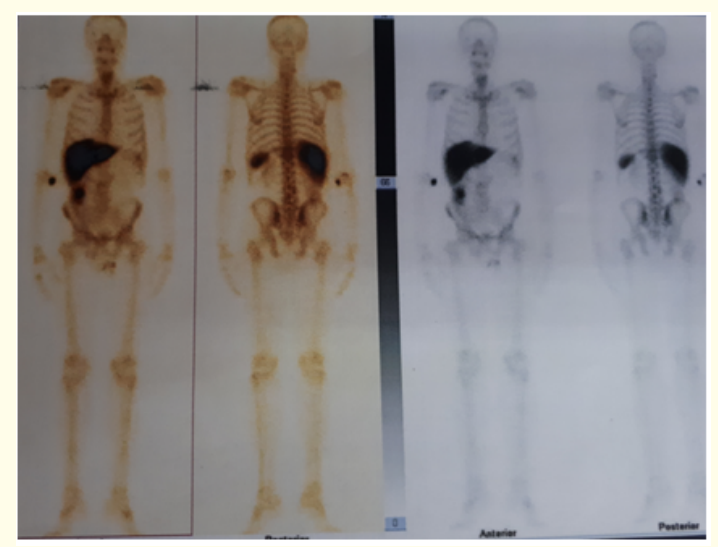

Figure 2: Pat. with Ca pullmo.

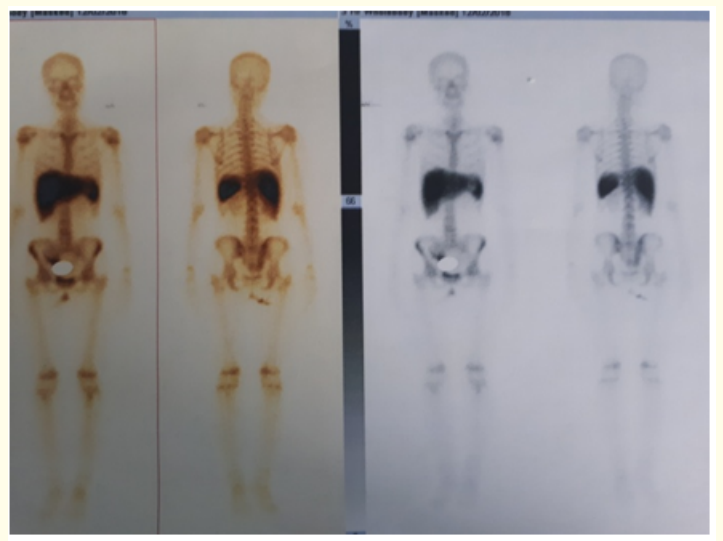

Figure 3: Three phase bone scan in patient with osteomyelitis suspp. 


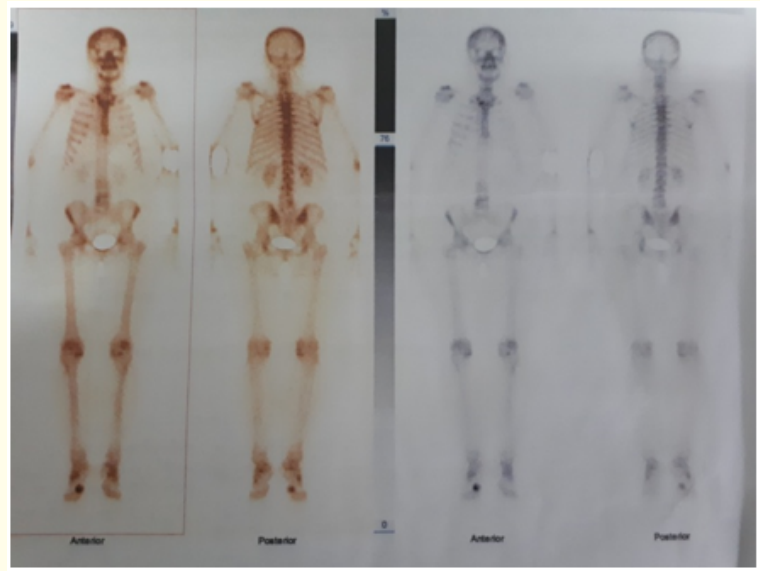

Figure 4

Since we did not have the ability to perform quality control, in the absence of material, in one of the three patients (the selection was done voluntarily) it was required to carry out the bone scan, in order to verify accumulation outside the bone tissue, other bone scanning has resulted with no artifacts, figure below. The patient suffers from breast cancer from 2013 and is under regular control.

\section{Discussion and Conclsuion}

The labeled preparation for these 3 patients was initially mitigated by $5 \mathrm{ml}$ physiological digestion, from which $3 \mathrm{ml}$ was labeled and the remaining $2 \mathrm{ml}$ were excreted on the next day, where along with the new ligand and the remaining $2 \mathrm{ml}$ of the MDP of the day previously, radiopharmaceutical labeling has been made for patients scheduled for the second day. None of these patients had any artifacts registered in the bone scan. It was questioned for a medical technician's verification in charge of preparation of radiopharmaceutical preparations for that day in a "hot" laboratory to verify the means used for labeling the radio-pharmaceutical preparation, and found that there was no make a technical mistake, but we found that the amount from the second elution from the $99 \mathrm{mTc}$ generator was added to $99 \mathrm{mTc}$ pure with less activity left over from the morning eluting, with the aim of achieving sufficient activity for performing three bone scans of the last patients, who had resulted in all three with intense diffuse accumulation in the liver and spleen, in addition to the usual accumulation on the skeleton.

Since the same radiopharmaceuticals used in 3 patients with liver and spleen artifacts $(2 \mathrm{ml}$ of the remaining amount of remain- ing radiopharmaceutical), were used in the following days for the same purposes, where none of the patients had shown abnormal accumulation outside the bone tissue excludes the possibility of oxidation during storage and preparation of the kit as a factor that would contribute to reducing the reduction capacity of $\operatorname{Sn} 2$, which usually fails to lower the $99 \mathrm{mTc}$ valence to suspect the condition of $99 \mathrm{mTc}$ reduced, which is chemically more reactive and forms complexes such as methylphenidate MDP, on the other hand, none of the patients were in dialysis to suspect any contamination from the apparatus during hemodialysis with $\mathrm{Al} 3$, none were with any therapy that would create interacting complexes with the preparation, and not suffering from any liver disease, etc., and the fact itself and that these artifacts have been detected in three patients at the same time, the question is raised about the cause of a miscarriage of error [1-9].

\section{Our impression and thought}

We suspect that perhaps the insufficient drying of the column due to the early elusion (almost 2 hours after the first elution time), where on the tip of the needle it was possible to have an excessive amount of $\mathrm{Al} 3$, with higher concentrations of $\mathrm{Al} 3(10 \mu \mathrm{g} / \mathrm{mL})$ can react with $99 \mathrm{mTc}$-MDP that form solid particles taken from RES cells in the liver and spleen until lower concentrations of $\mathrm{Al} 3$ (1 g/ $\mathrm{mL}$ ) would block capillaries in the lungs.

We bring evidence of the radiopharmaceutical kit used by the licensed, termed and normal compound supplier, and bone scan images of 3 patients with 99mTc-MDP liver and spleen diffuse accumulation, an control image of the bone scan of one patient, which confirms that the artifact discovered early in the liver and spleen is now missing, in the inability to verify the quality control the prepared ligand with HPLC, therefore for this presentation with this nature of these artifacts, we expect opinions from relevant authors.

\section{Bibliography}

1. Couper I and Driver N. "Quality Assurance". In: Allwood AC, Fell JT, eds. Textbook of Hospital Pharmacy. Oxford: Blackwell Scientific Publications (1980).

2. "Quality Assurance of Radiopharmaceuticals. Report of a Joint Working Party: The UK Radiopharmacists Group and the NHS Pharmaceutical QC Committee". Nuclear Medicine Communications 22 (2001): 909-916.

3. James Doherty and David Graham. "The Radiopharmacy". 
4. Shankar Vallabhajosula., et al. "Altered Biodistribution of Radiopharmaceuticals: Role of Radiochemical/Pharmaceutical Purity, Physiological, and Pharmacologic Factors". Seminars in Nuclear Medicine 40.4 (2010): 220-241.

5. Hladik WB and Norenberg JP. "Problems associated with the clinical use of radiopharma-ceuticals: A proposed classification system and trouble shooting guide". European Journal of Nuclear Medicine 23 (1996): 997-1002.

6. Lentle BC., et al. "Latrogenic alterations in radionuclide biodistributions". Seminars in Nuclear Medicine 9 (1979): 131-143.

7. Hung JC., et al. "Radiopharmaceutical-related pitfalls and artifacts". Seminars in Nuclear Medicine 26 (1996): 208-255.

8. Hojelse C., et al. Factors which affect the integrity of radiopharmaceuticals, in Sampson CB (ed): Textbook of Radiopharmacy, Theory and Practice (ed 2). Amsterdam, Gordon and Breach Science Publishers (1994): 145-152.

9. 2010-2016 International Atomic Energy Agency (IAEA). Virtual Course in Radiopharmacy; Product issues with eluted $99 \mathrm{mTc}$.

\section{Volume 5 Issue 10 October 2021}

(C) All rights are reserved by Luljeta Abdullahu., et al. 